\title{
Factors Inducing Drainage Tube Complications After Hepatobiliary Surgery
}

\author{
Xuanjun Wang* \\ Changshu No. 2 People's Hospital, Changshu 215500, Jiangsu Province, China \\ *Corresponding author: Xuanjun Wang, 254839566@qq.com
}

Copyright: ( $) 2022$ Author(s). This is an open-access article distributed under the terms of the Creative Commons Attribution License (CC BY 4.0), permitting distribution and reproduction in any medium, provided the original work is cited.

\begin{abstract}
Objective: To explore the main factors of drainage tube complications after hepatobiliary surgery. Methods: From November 2019 to October 2021, 103 patients with drainage tube complications after hepatobiliary surgery in Changshu No. 2 People's Hospital were selected as subjects for this study; the factors of postoperative drainage tube complications were analyzed by retrospective analysis. Results: The complications of drainage tubes include cavity organ damage, sliding of drainage tube into the abdominal cavity, broken drainage tube, blocked drainage tube, bleeding in drainage tube, bleeding from the mouth of drainage tube, abdominal cavity infection caused by drainage tube, and intestinal obstruction caused by drainage tube compression; the number of cases were $9,8,12,21,18,17,8$, and 10 , accounting for $8.74 \%, 7.77 \%, 11.65 \%$, $20.39 \%, 17.48 \%, 16.50 \%, 7.77 \%$, and $9.70 \%$, respectively; the causes of these complications include early and late removal of drainage tube, improper positioning, color of drainage fluid, drainage tube falling out or self-removal, and so on. Conclusion: After hepatobiliary surgery, although the complications caused by drainage tubes have certain relationship with the indwelling time and surgery, the most critical is related to postoperative nursing care; therefore, it is necessary to observe the condition of the drainage tube and draining fluid after surgery, including the color of the fluid, its flow rate, and whether the drainage tube leaks or falls out; after surgery, patients should be encouraged to cooperate with the medical staffs, and family members should be reminded to pay attention to the observation of patients and informed about matters needing attention, so as to reduce the incidence of drainage tube complications after hepatobiliary surgery.
\end{abstract}

Keywords: Hepatobiliary surgery; Drainage tube complications; Postoperative care

Online publication: January 19, 2022

\section{Introduction}

As the largest internal organ of the human body, the liver participates in a number of vital functions necessary for human survival, such as the synthesis and secretion of bile ${ }^{[1]}$, the basic metabolic needs of the body, excretion and detoxification, phagocytosis, hematopoiesis, regeneration, etc. ${ }^{[2]}$. It is one of the important organs of the human body. The structure of the liver is complex, and the incidence of disease is also high. The types of diseases that require hepatobiliary surgery have brought many treatment issues. At present, common benign hepatobiliary diseases in China include gallstones, hepatobiliary stones, etc.; however, recurrent stone obstruction would result in cholangitis. Long-term recurrent cholangitis can cause liver fibrosis, imbalance liver morphology, portal hypertension, and even bile duct cancerization, which has become an important cause of death of benign biliary diseases in China ${ }^{[3-5]}$.

Hepatobiliary surgery began in 1888. Langenbuch, a German doctor, was the first to successfully carry out hepatectomy (left lobe hepatectomy). In 1978, Philipe Mouret, a gynecologist and obstetrician, carried out the world's first laparoscopic cholecystectomy, which marked the birth and development of minimally 
invasive (laparoscopic) surgery and promoted the development of hepatobiliary surgery. However, the development of hepatobiliary surgery technology has not prevented surgical complications from occurring. Due to its own anatomical structure and physiological characteristics in addition to the difficulty of some procedures, hepatobiliary surgery does not only have the universality of various complications of general surgery, but also its own uniqueness. The incidence of surgical complications is high. Most of the complications are related to the condition of the hepatobiliary system, improper surgical methods, surgical techniques, and poor perioperative management ${ }^{[6]}$. Research has found that in addition to bleeding, bile duct injury, and liver failure, bile leakage is also one of the most common complications of hepatobiliary surgery ${ }^{[7]}$, of which among all the complications, bile leakage is one of the most common causes of death [8].

\section{Materials and methods}

\subsection{General information}

From November 2019 to October 2021, 103 patients with drainage tube complications after hepatobiliary surgery were treated in Changshu No. 2 People's Hospital. The had an average age of 46 years; 53 were male patients and 50 were female patients; 46 patients had a smoking history and their weight ranged from $41 \mathrm{~kg}$ to $98 \mathrm{~kg}$. The clinical data of all the patients were complete, and drainage tubes were placed after hepatobiliary surgery. The patients signed the informed consent form prior to the study.

\subsection{Method}

Through retrospective analysis, the number of patients with drainage tube complications, such as infection, bile leakage, biliary bleeding, and other complications, after hepatobiliary surgery in Changshu No. 2 People's Hospital was determined. The number, causes, types, and probability of complications were statistically sorted out along with the measures that could be taken for different complications.

\section{Results}

\subsection{Statistics of complications}

Upon regression analysis, it can be appreciated that from the 103 patients admitted, the complications of drainage tube include cavity organ damage, sliding of drainage tube into the abdominal cavity, broken drainage tube, blocked drainage tube, bleeding in drainage tube, bleeding from the mouth of drainage tube, abdominal cavity infection caused by drainage tube, as well as intestinal obstruction caused by drainage tube compression. The number of cases were $9,8,12,21,18,17,8$, and 10 accounting for $8.74 \%, 7.77 \%$, $11.65 \%, 20.39 \%, 17.48 \%, 16.50 \%, 7.77 \%$, and $9.70 \%$, respectively, as shown in Table 1.

Table 1. Statistics of complications

\begin{tabular}{ccc}
\hline Types of complications & Number of cases & Proportion of complications (\%) \\
\hline Cavity organ damage & 9 & $8.74 \%$ \\
Sliding of drainage tube into the abdominal cavity & 8 & $7.77 \%$ \\
Broken drainage tube & 12 & $11.65 \%$ \\
Blocked drainage tube & 21 & $20.39 \%$ \\
Bleeding in drainage tube & 18 & $17.48 \%$ \\
Bleeding from the mouth of drainage tube & 17 & $16.50 \%$ \\
Abdominal infection caused by drainage tube & 8 & $7.77 \%$ \\
Intestinal obstruction caused by drainage tube compression & 10 & $9.70 \%$ \\
\hline
\end{tabular}




\subsection{Multiple factor analysis}

Taking specific complications as dependent variables, regression analysis was carried out. There were five main causes for these complications: early removal, late removal, improper positioning, color of drainage fluid, as well as the falling out and self-removal of drainage tube. The $95 \%$ confidence intervals were 0.55 1.94, 0.46-8.52, 1.22-7.95, 0.48-2.26, and 3.34-13.81, respectively. Among them, drainage tube failing out or self-removal and improper positioning of drainage tube are independent risk factors.

\section{Discussion}

Postoperative short-term indwelling drainage tube is commonly seen in hepatobiliary surgery, but it also carries risk of complications. Research has shown that cavity organ injury, bleeding, sliding of drainage tube into the abdominal cavity, abdominal infection, and drainage port infection caused by drainage tube account for high proportions ${ }^{[2]}$. Therefore, analyzing the causes of drainage tube complications is of great significance to prevent complications during the retention period of drainage tubes. The results of this study showed that the main causes are drainage tube indwelling time, drainage tube falling out or self-removal shortly after surgery, positioning of drainage tube, and drainage fluid color. Multivariate logistic regression analysis showed that drainage tube falling out or self-removal shortly after surgery and improper positioning of drainage tube are independent risk factors of postoperative drainage tube complications ${ }^{[3]}$.

Several studies have shown that during the indwelling of drainage tube, if the drainage period is insufficient or if the drainage tube is removed in advance, the drainage of bile from the gallbladder would be inadequate, thus leading to biliary leakage or recurrent symptoms. According to a study by Yingqin Zhang and other researchers, some patients still experience inflammation one week after hepatobiliary surgery, and some even have severe hydrocholecystis and adhesion. Therefore, the falling out or selfremoval of drainage tube shortly after surgery would result in poor drainage of inflammatory secretion from the gallbladder ${ }^{[4]}$. In this study, $58.8 \%$ of the patients had complications involving the abdomen. According to analysis, the reason may be due to the diffusion of cholecystitis secretion along the gallbladder fistula ${ }^{[5]}$. Therefore, in clinical diagnosis and treatment, the nursing care for drainage tubes should be strengthened, the patients should be encouraged to strictly abide by the doctor's advice, and they should not remove the tube on their own. At the same time, when placing the drainage tube, the tube should be reasonably positioned as far as possible to prevent the drainage tube from falling out by itself. The results of this study showed that improper positioning is another independent risk factor of drainage tube complications. Previous studies have shown that improper positioning would lead to infection, biliary bleeding, obstruction, and other problems. If it is not handled in time or in an incorrect manner, there may be serious consequences; this is not conducive to the postoperative recovery of general surgery patients. Therefore, it is necessary to reinforce the observation of postoperative drainage tubes, deal with drainage tube abnormalities as soon as possible, change the dressing frequently, and disinfect the site well ${ }^{[6]}$.

Prior to hepatobiliary surgery, patients need to evaluate their own risk factors. For patients with poor physique or diseases, nutritional support should be provided before the procedure. Preoperative intensive training and multichannel training should be carried out, and the indications of various hepatobiliary surgery methods should be well understood before surgery. Intraoperative cholangiography should be performed routinely during the initial stage of laparoscopic cholecystectomy (LC) in primary hospitals and in the first 100 to 200 cases taken up by the operator to avoid iatrogenic bile duct injury and choledocholithiasis residue. After being proficient in the technique, intraoperative cholangiography can be performed selectively. Perioperative nursing and hepatobiliary surgery education should be emphasized ${ }^{[5,8]}$. In conclusion, after hepatobiliary surgery, although the complications caused by drainage tube have a certain relationship with the indwelling time and surgery, the most critical is related to postoperative nursing care. It is necessary to observe the condition of the drainage tube after surgery: whether the drainage tube 
leaks or falls out. In addition, it is necessary to observe the fluid from the drainage tube, including its color and flow rate. After surgery, patients should be encouraged to cooperate with the medical staffs, and family members should be reminded to pay attention to the observation of patients and informed about matters that need attention, so as to reduce the incidence of drainage tube complications after hepatobiliary surgery.

\section{Disclosure statement}

The author declares that there is no conflict of interest.

\section{References}

[1] Fang Y, Dai G, Shen S, 2021, Analysis of the Efficacy and Complications of Precise Hepatic Resection based on Anatomic Hepatectomy for Hepatobiliary Malignancy. Chinese And Foreign Medical Research, 19(30): 172-174.

[2] Zhong F, Gan H, Zhang C, 2021, Effect of Medical Failure Mode and Effect Analysis on Postoperative T-type Drainage Management of Patients undergoing Hepatobiliary Surgery in General Surgery. International Journal of Nursing, 40(12): 2219-2222.

[3] Chen S, Wang L, Mi Y, et al., 2021, Diagnosis, Treatment and Prevention of Biliary Leakage Complications after Percutaneous Hepatolithiasis. Journal of Hepatopancreatobiliary Surgery, 33(06): 362-364.

[4] Zhang Y, Liu H, Tan X, 2021, Effect of Cognitive Intervention on Health Education After Percutaneous Hepatobiliary Drainage. China Continuing Medical Education, 13(11): 192-195.

[5] Wang J, 2020, Effect of Minimally Invasive Treatment on Improving the Therapeutic Effect of Hepatobiliary Surgery. Renowned Doctor, 2020(07): 28-29.

[6] Song H, 2020, Observation on Clinical Application and Curative Effect of Damage Control Surgery in Hepatobiliary Surgery. Health for Everyone, 2020(04): 83.

[7] Shen Y, Sun X, 2019, Effect Research of Postoperative Rehabilitation Measures on Recovery of Patients After Hepatobiliary Surgery. Chongqing Medical, 48(12): 2127-2129.

[8] Chai N, 2019, Discussion on Cause Analysis and Treatment Strategy of Bile Leakage after Hepatobiliary Surgery. Lanzhou University. 